Check for updates

Cite this: Mater. Adv., 2020, 1,3556

Received 13th April 2020, Accepted 15th October 2020

DOI: 10.1039/d0ma00199f

rsc.li/materials-advances

\title{
Cytotoxicity induced by new spiral mesoporous silica nanorods via specific surface area and ROS accumulation in HeLa cells
}

\author{
Lan She, iD a Miao Sun, ${ }^{a}$ Xinfang Li, ${ }^{a}$ Anfeng Kang, ${ }^{a}$ Feng Yang, ${ }^{a}$ Yonghui Deng, (DD b \\ Dan Wang ${ }^{c}$ and Xinrong Zhang*a
}

\begin{abstract}
Mesoporous silica nanoparticles (MSNs), with good biological safety and drug carrying capacity, are being widely used for applications in biomedical research. In this study, three new spiral mesoporous silica nanorods (MSNRs) with different aspect ratios were synthesized through adjusting the dosage of $\mathrm{NH}_{3} \cdot \mathrm{H}_{2} \mathrm{O}$ by the modified Stöber method. Furthermore, MSNRs were used to study their interactions with HeLa cells. The synthesized MSNRs had periodical thread and narrower aperture size distribution. As indicated by laser scanning confocal microscopy (LSCM) and biological transmission electron microscopy, MSNRs were found to be internalized by HeLa cells and were primarily localized in the cytoplasm, lysosomes and membranous vesicles. When the concentration of MSNRs reached $320 \mu \mathrm{g} \mathrm{mL}^{-1}, \mathrm{MSNRs}$ had different degrees of cytotoxicity. The cytotoxicity was positively correlated with the size of the specific surface area of MSNRs. Reactive oxygen species (ROS) production was induced in the HeLa cells by the MSNRs, and reactive oxygen species generation might be the major factor causing cytotoxicity. The results of this study indicated that the effect of MSNRs on HeLa cell viability and cellular oxidative stress was related to specific surface area and has great potential in drug loading and delivery.
\end{abstract}

\section{Introduction}

In recent years, a variety of nanomaterials, such as mesoporous silica nanoparticles (MSNs), graphene, quantum dots (QDs) and carbon nanotubes (CNTs) have been synthesized and applied in biomedical research. ${ }^{1,2}$ As is well known, nanoparticles exhibit medical potential because of their unique physicochemical and biological features, including high specific surface area, specific structural properties, ability to carry drugs, capability to cross cell barriers and long circulation time in blood. ${ }^{3-5}$ MSNs, as a kind of non-metal oxide, has been extensively used in biomedicine, pharmaceuticals and other industrial manufacturing fields, such as cancer therapy, biosensors, and drug delivery. ${ }^{6-8}$ Moreover, MSNs have drawn much attention as promising drug carriers in controlled drug delivery since they biodegrade in a controlled manner. ${ }^{9}$ In particular, biodegradable mesoporous silica has been used in drug carriers to store unstable proteins or poorly soluble drugs

\footnotetext{
${ }^{a}$ Department of Inorganic Chemistry, School of Pharmacy, Second Military Medical University, 325 Guohe Road, Shanghai 200433, China. E-mail: zhxr126@163.com ${ }^{b}$ Department of Chemistry, State Key Laboratory of Molecular Engineering of Polymers, Shanghai Key Laboratory of Molecular Catalysis and Innovative Materials, iChEM, Fudan University, Shanghai, 200433, China ${ }^{c}$ Department of Gynaecology and Obstetrics, Changzheng Hospital, Second Military Medical University, Shanghai, 200003, China
}

for sustained and controlled delivery to target tissues. ${ }^{10-12}$ In the synthesis process of MSNs by the modified Stöber method, ${ }^{13}$ a new type of spiral mesoporous silica nanorods (MSNRs) with different aspect ratios was synthesized by adjusting the dosage of $\mathrm{NH}_{3} \cdot \mathrm{H}_{2} \mathrm{O} \cdot{ }^{14,15}$ Interestingly, a recent study reported that the "rotational hopping" movement of nanorod particles can promote its diffusion speed in the grid like mucin fluid; therefore, nanorods can effectively improve the efficiency of drug delivery. ${ }^{16}$ The MSNRs fabricated by our method possessed the characteristics of large pore volume and specific surface area, and had potential as a new drug carrier.

At present, a number of studies on the cytotoxicity of MSNs have been performed to explore their biocompatibility using various human cell types such as the MDA-MB-231 breast cancer cells, ${ }^{17}$ Ovcar8-IP cells ${ }^{18}$ and A549 cells. ${ }^{19}$ However, there are few reports on the potential toxic impact of MSNRs. The present study aimed to synthesize new spiral MSNRs and investigate the effect of MSNRs in HeLa cells, which probably implied the possibility of MSNs being widely used in drug loading and delivery.

\section{Experimental}

The HeLa tumor-cell line, a human cervical cancer cell line originating from an American woman, Henrietta Lacks, was 
established in $1951 .^{20}$ This cell line has been widely used in cytotoxicity, tumor research and biological experiments in the medical field. ${ }^{21-23}$ The morphology of the MSNRs was verified by field emission scanning electron microscopy (FESEM) and transmission electron microscopy (TEM). The specific surface area, pore volume, and pore size of MSNRs were measured based on $\mathrm{N}_{2}$ adsorption/desorption isotherms using a pore size analyzer. The XRD patterns of the samples were analyzed through an X-ray diffractometer. The interaction between MSNRs and HeLa cells was explored. Laser scanning confocal microscopy (LSCM) was used to study the uptake of MSNRs in HeLa cells. Cell ultrastructure was detected by biological TEM. Cell viability and oxidative stress level were detected to access the cytotoxicity of MSNRs.

\subsection{Materials and methods}

RPMI-1640 medium and fetal bovine serum (FBS) were purchased from Gibco, USA. Cell counting kit (CCK-8) was obtained from Dojindo, Chemical Co., Ltd (Japan). Unless otherwise specified, the reagents and chemicals were purchased from Sinopharm Chemical Reagent Co., Ltd (Shanghai, China). Phosphate buffer saline (PBS) and 0.25\% trypsin enzyme solution were purchased from Bio-light Biotech (Shanghai, China). Reactive oxygen species assay kit (ROS) was purchased from Beyotime Biotechnology Research Institute (Jiangsu, China). Fluorescein isothiocyanate (FITC) and DAPI were obtained from Sigma Aldrich (St. Louis, USA). Laboratory consumables and equipment were purchased from Thermo Fisher Scientific (Shanghai, China). HeLa cells were presented by the National Key Laboratory of Medical Immunology, the Second Military Medical University.

\subsection{Preparation of MSNRs}

MSNRs were prepared in a Stöber solution (containing CTAB, TEOS, $\mathrm{NH}_{3} \cdot \mathrm{H}_{2} \mathrm{O}$, and deionized water) by a modified surfactant assembly sol-gel method. ${ }^{24,25}$ Briefly, $1.0 \mathrm{~g}$ CTAB was dissolved in $160 \mathrm{~mL}$ of deionized water at $35{ }^{\circ} \mathrm{C}$. After $15 \mathrm{~min}, 10 \mathrm{~mL}$, $8 \mathrm{~mL}$ and $4 \mathrm{~mL}$ of $\mathrm{NH}_{3} \cdot \mathrm{H}_{2} \mathrm{O}$ were added dropwise into the system, respectively. In addition, TEOS ( $5 \mathrm{~mL}$ ) was added slowly and uniformly into the solution under stirring. After stirring for $12 \mathrm{~h}$, the product was collected by centrifugation (5000 rpm, and $10 \mathrm{~min}$ ), then washed three times with water and ethanol. After that, the white products (silica-CTAB composites) were dispersed in ethanol solution $(100 \mathrm{~mL})$ containing concentrated $\mathrm{HCl}$ $(1.0 \mathrm{~mL})$ and stirred at $90{ }^{\circ} \mathrm{C}$ for $5 \mathrm{~h}$ to remove the template CTAB. The surfactant extraction process was repeated two times to ensure complete removal of CTAB. Next, the products were washed by deionized water three times and MSNRs were obtained after vacuum drying. Three different morphologies of MSNRs were prepared by adjusting the dosages of $\mathrm{NH}_{3} \cdot \mathrm{H}_{2} \mathrm{O}$. These particles were denoted as MSNR1, MSNR2 and MSNR3 according to the $\mathrm{NH}_{3} \cdot \mathrm{H}_{2} \mathrm{O}$ dosage. FITC labeled MSNRs were synthesized simultaneously for further experiments.

\subsection{Characterization of MSNRs}

The morphology and physicochemical properties of the MSNRs were verified by field emission scanning electron microscopy (FESEM, S4800, Hitachi, Japan) and transmission electron microscopy (TEM, JEM2010, JEOL, Japan). The average lengths of MSNRs were estimated from cross-sectional FESEM images. The specific surface area, pore volume, and pore size of MSNRs were measured based on $\mathrm{N}_{2}$ adsorption/desorption isotherms using a pore size analyzer (TriStar 3000, GA, USA). The measurement was conducted under a nitrogen atmosphere with the weight of each sample set at $2 \mathrm{~g}$. The XRD patterns of the samples were analyzed through an X-ray diffractometer (XRD, D4, Bruker, Germany). The pore size and distribution of the MSNRs were verified through the Barrett-Joyner-Halanda $(\mathrm{BJH})$ method. The size and diameter distribution of MSNRs micelles were measured by dynamic light scattering (DSL, ZEN3600, Malvern Panalytical, UK). Additionally, fabrication was verified by Fourier transform infared spectroscopy (FTIR) analysis (Nexus Model 470; GMI, Ramsey, MN, USA) in the range of $400-4000 \mathrm{~cm}^{-1}$.

\subsection{Cell culture and treatment}

After recovery, HeLa cells were grown in a $25 \mathrm{~cm}^{2}$ cell culture flask at $37{ }^{\circ} \mathrm{C}$ under $5 \% \mathrm{CO}_{2}$ atmosphere using Roswell Park Memorial Institute (RPMI)-1640 culture medium containing $10 \%$ fetal bovine serum, and 1\% penicillin/streptomycin. For splitting and seeding, the HeLa cells were detached from the flask with $0.25 \%$ trypsin-EDTA.

\subsection{Laser scanning confocal microscopy}

A total of $1 \times 10^{5}$ HeLa cells were seeded into $35 \mathrm{~mm}$ glass bottom dishes for laser scanning confocal microscopy (LSCM, Leica TCS SP2, Solms, Germany). After incubating for $12 \mathrm{~h}$, the medium in each dish was removed and $15 \mu \mathrm{g} \mathrm{mL}^{-1}$ FITCMSNRs in RPMI-1640 medium were added to the plates. Subsequently, the cells were incubated for $2 \mathrm{~h}$ at $37{ }^{\circ} \mathrm{C}$ in an incubator containing $5 \% \mathrm{CO}_{2}$. Then HeLa cells in each dish were washed by PBS three times, and the cells were fixed by $1 \mathrm{~mL}$ of $4 \%$ paraformaldehyde for $20 \mathrm{~min}$. Once fixed, the samples were washed thrice with PBS and stained by 4',6diamidinio-2-phenylindole (DAPI) for $20 \mathrm{~min}$. After washing with PBS, the HeLa cells were observed by LSCM. The falsecolored images were obtained with an excitation wavelength of $358 \mathrm{~nm}$, and emission fluorescence was collected at a wavelength of $488 \mathrm{~nm}$.

\subsection{Biological transmission electron microscopy}

HeLa cells were incubated in a Petri dish (10 cm in diameter) until $80-90 \%$ of the surface of the culture dish was covered. After the culture medium was discarded, $8 \mathrm{~mL}$ of RPMI-1640 medium supplemented with MSNRs $\left(50 \mu \mathrm{g} \mathrm{mL}{ }^{-1}\right)$ was added into the culture dish and kept incubating for $4 \mathrm{~h}$, and the cells were washed thrice with PBS and collected after centrifugation at $1000 \mathrm{rpm}$ for $5 \mathrm{~min}$. After fixing the cells with the prepared cellular fixture $\left(2.5 \%\right.$ glutaraldehyde), samples were kept at $4{ }^{\circ} \mathrm{C}$ for 4-6 h. The ultrastructure images of HeLa cells were obtained using a biological TEM (H-7650, Hitachi, Japan).

\subsection{Cell viability}

In vitro cell viability was accessed using the cell counting kit-8 (CCK-8) method, which was widely used to evaluate cytotoxicity. 
The cell viability was detected according to the manufacturer's instruction. HeLa cells were seeded in 96-well flat-bottomed plates with a density of $5 \times 10^{3}$ cells per well, then incubated in a $\mathrm{CO}_{2}$ atmosphere at $37{ }^{\circ} \mathrm{C}$ for $12 \mathrm{~h}$. Next, the medium in each well was removed and MSNRs $(100 \mu \mathrm{L})$ with various concentrations in RPMI-1640 medium were added to the wells. Subsequently, the cells were incubated for $24 \mathrm{~h}$. Next, the solution in each well was removed and $100 \mu \mathrm{L} 10 \%$ CCK-8 solutions were added to each well. After incubation for $2 \mathrm{~h}$, the absorbance was measured at $450 \mathrm{~nm}$ using a microplate reader (BioTek USA). The relative survival rate of HeLa cells was calculated using the following formula:

$$
R(\%)=\left(A_{\text {sample }}-A_{\text {blank }}\right) /\left(A_{\text {negative }}-A_{\text {blank }}\right) \times 100 \%
$$

\subsection{Oxidative stress}

The ROS measurement was used to indicate the oxidative stress caused by MSNRs. HeLa cells were seeded into 6-well plates with a density of $4 \times 10^{5}$ cells per well and incubated with different concentrations of MSNRs. Cells incubated with serum-free RPMI-1640 medium were used as a control group. After treatment for $4 \mathrm{~h}$, the cells were washed three times with PBS and then incubated with $10 \mu \mathrm{M}$ 2,7-dichlorodihydrofluorescein diacetate (DCFH-DA) for $20 \mathrm{~min}$ in the dark at $37{ }^{\circ} \mathrm{C}$. After washing with PBS, HeLa cells were collected and centrifuged at $1000 \mathrm{rpm}$ for $5 \mathrm{~min}$. Subsequently, the samples were suspended in $0.5 \mathrm{~mL}$ PBS and the fluorescent density was detected with flow cytometry (FACS, BD Biosciences, USA). The detection parameters were an excitation wavelength of $488 \mathrm{~nm}$ and an emission wavelength of $525 \mathrm{~nm}$.

\subsection{Statistical analysis}

All the experimental data were expressed as means \pm standard deviation (SD) from three separate experiments. Date were analyzed by one-way ANOVA followed by Dunnett's $t$-test for comparisons between groups, and $P<0.05$ indicated a statistically significant difference.

\section{Results and discussion}

\subsection{Characterization of MSNRs}

MSNRs were prepared according to the modified Stöber method and were characterized by FESEM and TEM (Fig. 1). The FESEM images showed that when the dosage of ammonia was $10 \mathrm{~mL}$, the mesoporous silica rods (MSNR1) had a clear and regular six square shape spiral morphology with a slight bulge at both ends and were more uniform in size (Fig. 1a and b). When the dosage of ammonia was $8 \mathrm{~mL}$, the mesoporous silica rods (MSNR2) still maintained the spiral shape. The length of the rods decreased, while the diameter and the helix degree increased. The rod size was more uniform with a better monodispersity (Fig. 1d and e). When the dosage of ammonia was $4 \mathrm{~mL}$, the length and the diameter of the mesoporous silica rods (MSNR3) were further shortened (Fig. $1 \mathrm{~h}$ and i). The TEM results showed that along the long axis of spiral MSNRs, at intervals of a certain distance, the mesoporous orifice showed a periodical twist, which indicated that the mesoporous channel was a spiral array coiled along the long axis. MSNR1 had a six square shaped spiral helical morphology (Fig. 1c), the pitch of MSNR2 was smaller and the direction of mesoporous path also presented periodic parallel mesoporous stripes, which indicates that the spiral channel of MSNRs was chiral (Fig. 1f) and the chirality of the spiral channel was formed by removing the chiral organic group in the preparation. ${ }^{26}$ The helix morphology of MSNR3 remained unchanged, while the ordering of mesoporous channels decreased (Fig. 1j). The length-diameter ratios were estimated from FESEM images. The results showed that the diameter of MSNR1 was the largest, while the ratio of length to diameter was the smallest (3.5). The draw ratios of MSNR2 and MSNR3 were close to 4.6 and 4.8, respectively.

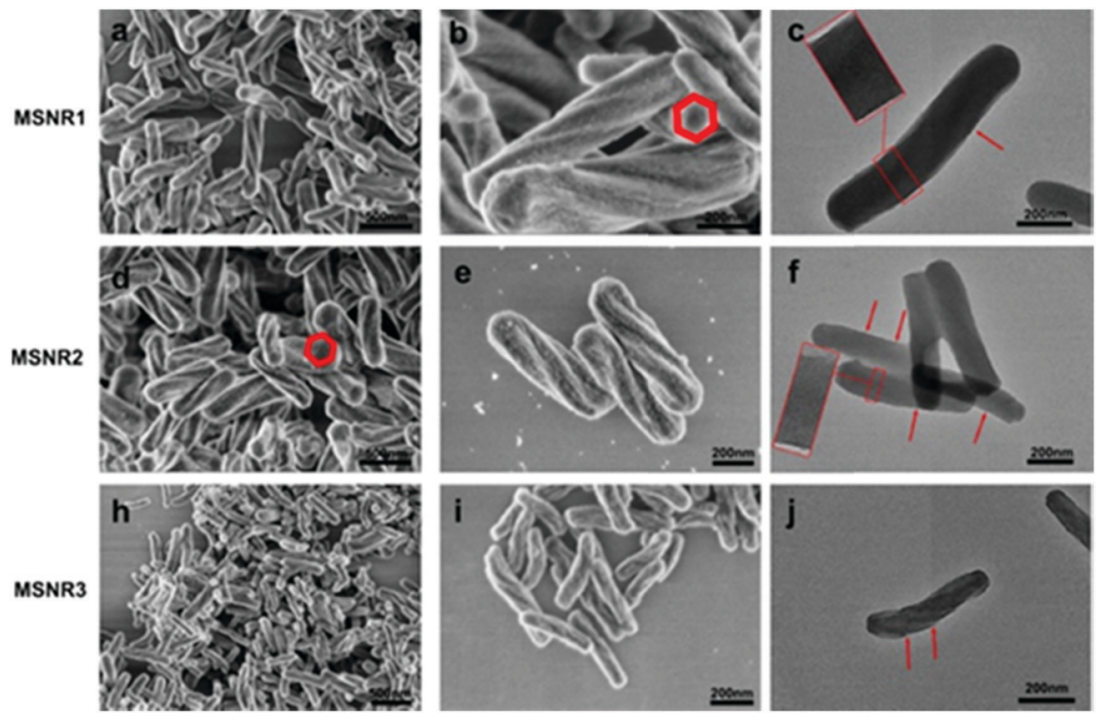

Fig. 1 Field emission scanning electron microscopy images ( $a, b, d, e, h$ and i) and transmission electron microscopy images (c, f and j) of MSNRs. 


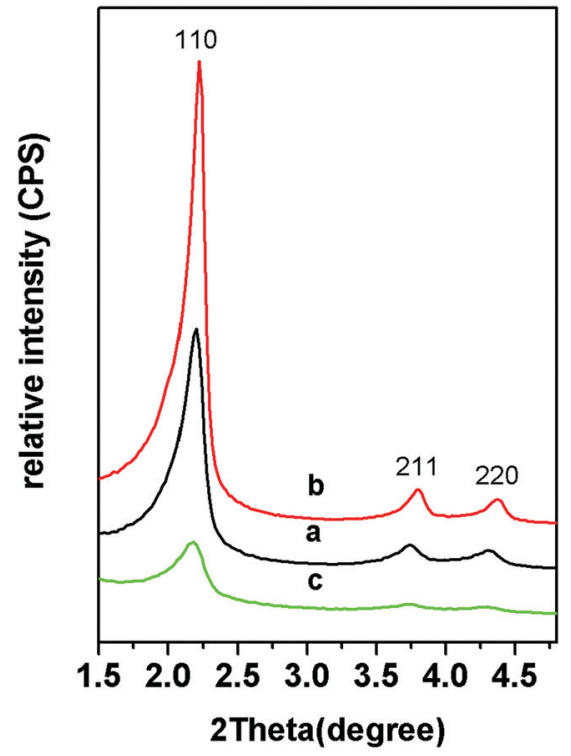

Fig. 2 Small-angle XRD patterns of MSNR1 (a), MSNR2 (b) and MSNR3 (c).

The small angle XRD patterns of the samples showed that there were three obvious diffraction peaks of MSNRs (Fig. 2). The ratio of the diffraction peak to the reciprocal of the spacing of the crystal plane $(d)$ was $1: \sqrt{ } 3: 2$; hence we determined that the mesoscopic structure of the samples was $p 6 \mathrm{~mm}$ and the three diffraction peaks corresponded to the (10), (11) and (20) sides of the $p 6 \mathrm{~mm}$ two-dimensional hexagonal mesostructure. When the usage of ammonia water was $8 \mathrm{~mL}$, the diffraction peaks of MSNR2 were relatively sharp (Fig. 2b), while when the ammonia water was $4 \mathrm{~mL}$, the diffraction peaks of MSNR3 were relatively wide (Fig. 2c), which indicated that the formation of the ordered mesoscopic structure was more favored with ammonia catalysis.

$\mathrm{N}_{2}$ adsorption/desorption isotherms of MSNRs are illustrated and summarized in Fig. 3 to investigate the surface
Table 1 Textural properties of MSNRs

\begin{tabular}{llll}
\hline & $\begin{array}{l}\text { BET surface } \\
\text { area }\left(\mathrm{m}^{2} \mathrm{~g}^{-1}\right)\end{array}$ & $\begin{array}{l}\text { Pore volume } \\
\left(\mathrm{cm}^{3} \mathrm{~g}^{-1}\right)\end{array}$ & $\begin{array}{l}\text { Pore size } \\
(\mathrm{nm})\end{array}$ \\
\hline MSNR-1 & 1092 & 0.99 & 2.78 \\
MSNR-2 & 1251 & 1.05 & 2.78 \\
MSNR-3 & 1055 & 0.97 & 2.94
\end{tabular}

area and pore size distribution. According to the six classified types of adsorption isotherms of porous powders, the MSNRs showed a typical IV type isotherm curve for mesoporous materials. In the range of $P / P_{0} 0.2-0.4$ (Fig. 3A), there was a sharp increase of adsorption caused by capillary condensation, indicating that the mesoporous distribution of MSNRs was very concentrated. The mean pore sizes of MSNR1, MSNR2 and MSNR3 were $2.78 \mathrm{~nm}, 2.78 \mathrm{~nm}$ and $2.94 \mathrm{~nm}$, respectively (Fig. 3B). The results of Table 1 showed that when the dosage of ammonia was $8 \mathrm{~mL}$, MSNR2 possessed the maximum specific surface area $\left(1251 \mathrm{~m}^{2} \mathrm{~g}^{-1}\right)$ and pore volume $\left(1.05 \mathrm{~cm}^{3} \mathrm{~g}^{-1}\right)$ among the three kinds of MSNRs.

TEM and EDAX results are shown in Fig. 4, with the corresponding $\mathrm{N}$ (c), Si (d), O (e) and $\mathrm{C}$ (f) elemental face mapping of MSNR2, indicating the high homogeneous dispersion of $\mathrm{N}$ and $\mathrm{C}$ in the silica matrix. In addition, the EDAX result only shows the $\mathrm{Si}, \mathrm{O}, \mathrm{N}$ and $\mathrm{C}$ signal, which further confirms the purity of the synthesized nanomaterials.

FTIR spectra evidenced the successful $-\mathrm{OH}$ modification on the surface of mesoporous silica (Fig. 5). In order to confirm that the-OH group was successfully introduced to the MSNR2, the FTIR spectra was collected, as shown in Fig. 5. From the FTIR spectra, it can be clearly seen that the MSNR2 exhibited typical related absorption bands at $3442 \mathrm{~cm}^{-1}$ and $1089 \mathrm{~cm}^{-1}$, corresponding to the $\mathrm{O}-\mathrm{H}$ stretching vibration and $\mathrm{Si}-\mathrm{O}$ skeleton stretching vibration, respectively, so the results of FTIR spectra showed that a large number of $\mathrm{Si}-\mathrm{OH}$ have been successfully loaded in the surface of MSNR2.
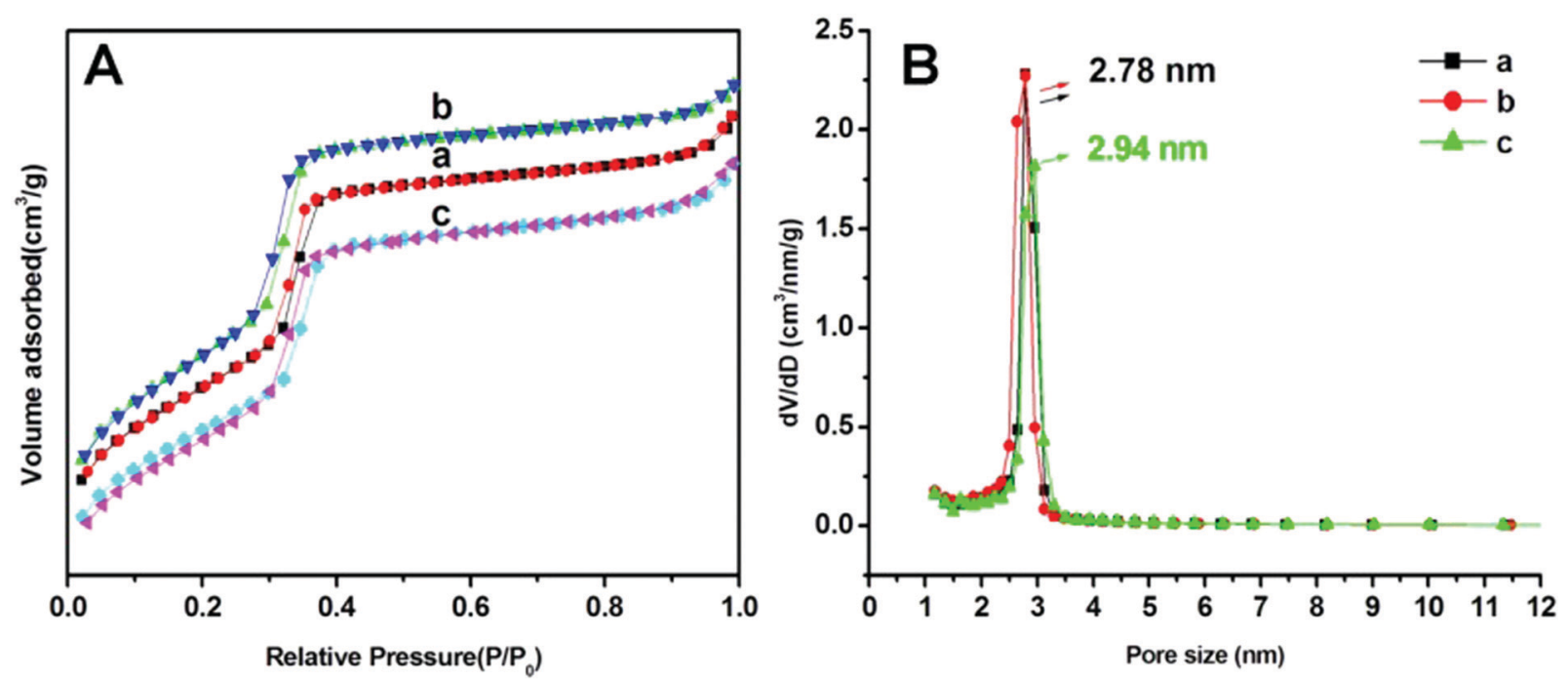

Fig. 3 Adsorption-desorption isotherms (A) and pore size distributions (B) of MSNR1 (a), MSNR2 (b), and MSNR3 (c). 

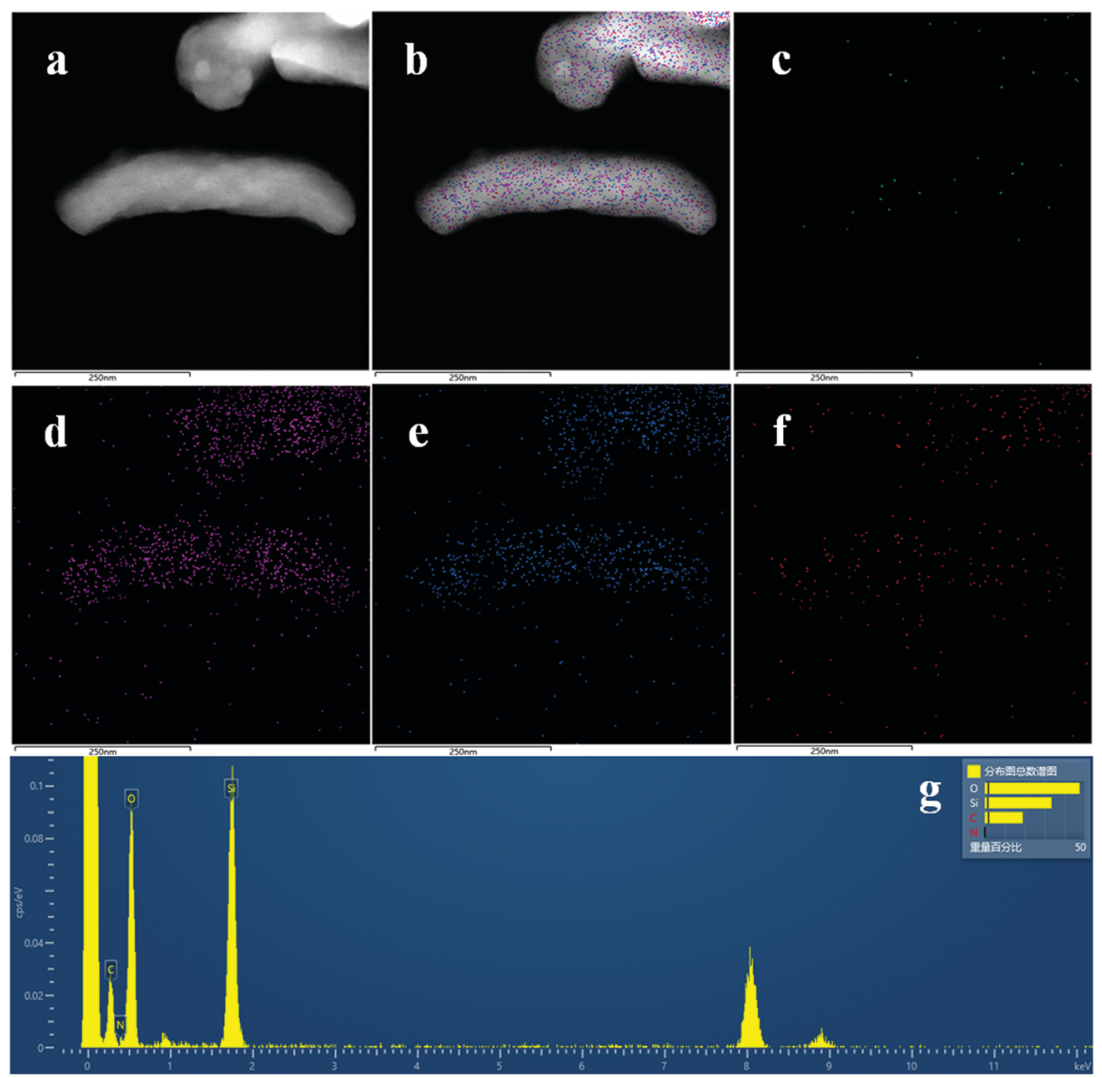

Fig. 4 TEM images of MSNR2 ( $a$ and b), elemental mapping of $N(c), S i(d), O(e)$ and $C$ ( $f$ ), respectively, and EDAX result (g).

Size and charge of mesoporous silica nanoparticles (MSNs) play a major role in the stability of the nanoparticles. As shown in Fig. 6, MSNR2 exhibited an average hydrodynamic diameter of $240 \mathrm{~nm}$ determined by dynamic light scattering (Fig. 6A). The surface zeta potential of MSNR2 was $-17.5 \mathrm{mV}$ due to charge screening of $-\mathrm{OH}$ (Fig. 6B). Also, the stability of the MSNR2 dispersion was estimated by PDI changes in $48 \mathrm{~h}$ after

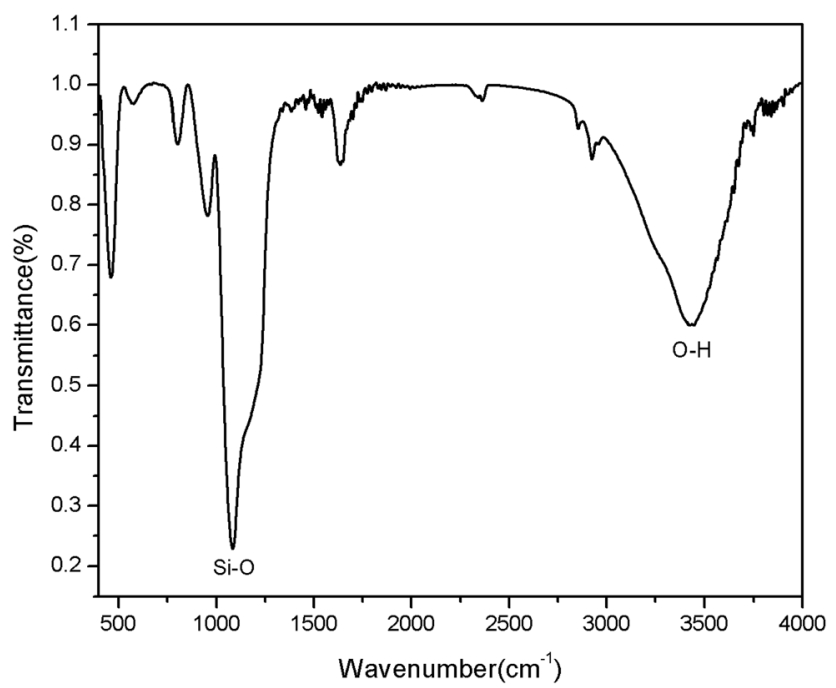

Fig. 5 FTIR spectra of MSNR2. dispersion under faintly acidic conditions, as shown in Fig. 7. The MSNR2 nanorods showed high stability and benign dispersity under faintly acidic conditions.

\subsection{Cellular uptake by LSCM}

Cellular uptake is essential for drug carriers in vivo. Fluorescence images of HeLa cells were obtained using LSCM, as shown in Fig. 8. DAPI (blue in Fig. 8) stained the DNA of living cells and FITC (green in Fig. 8) was used to mark the location relationship between MSNRs and HeLa cells. The results showed that MSNRs could be taken by endocytosis and distributed in the cytoplasm of HeLa cells, which could be used as potential drug carriers to achieve antitumor therapy.

\subsection{The ultrastructure of HeLa cells}

Internalization and intracellular distribution of the MSNRs in HeLa cells were investigated by biological TEM as shown in Fig. 9. TEM images showed that the three types of MSNRs mainly endocytosed HeLa cells by phagocytosis. Except for small single particles in the lysosomes and cytoplasm, MSNRs were present in lysosomes and membranous vesicles in the form of aggregates. It was noteworthy that MSNRs were not observed in the nucleus.

\subsection{Cell viability}

HeLa cells were exposed to a range of concentrations of MSNRs $\left(625,312.5,156.25,78.125,39.06,19.5,9.75\right.$ and $\left.4.825 \mu \mathrm{gL}^{-1}\right)$ 

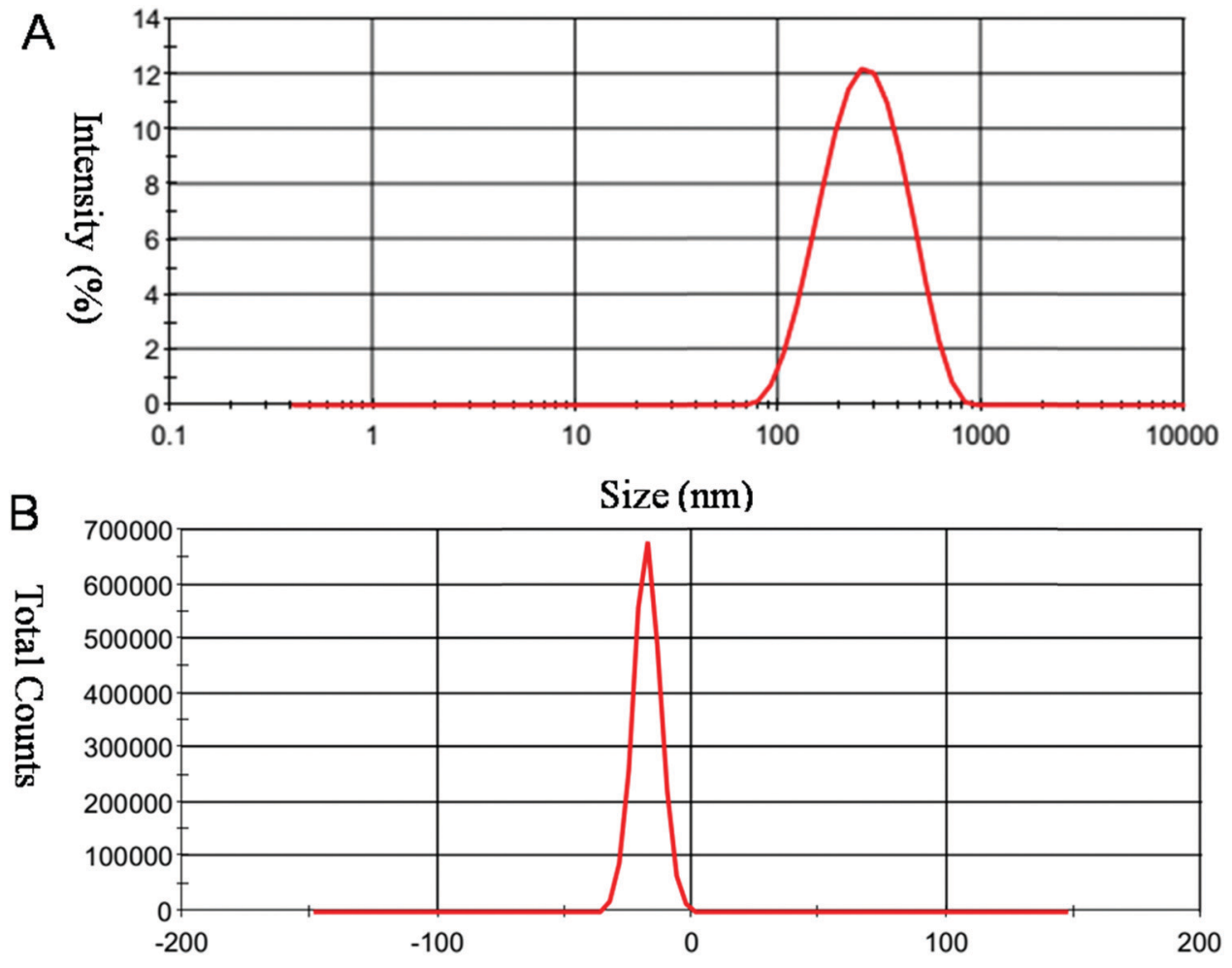

Zeta Potential (mV)

Fig. 6 Typical DLS profile of MSNR2 measured in aqueous solution. Notes: (A) hydrodynamic diameters. (B) Zeta potential measurements of nanoparticles.

for $24 \mathrm{~h}$. The cell viability was assessed by the CCK-8 method and the result was presented as a percentage of control group viability (Fig. 10). $\mathrm{IC}_{50}$ values were calculated as MSNR1 (300.2 $\left.\mu \mathrm{g} \mathrm{mL}^{-1}\right)$, MSNR2 (128.3 $\left.\mu \mathrm{g} \mathrm{mL}^{-1}\right)$, and MSNR3 (306.4 $\left.\mu \mathrm{g} \mathrm{mL} \mathrm{m}^{-1}\right)$. When the concentration of MSNRs was

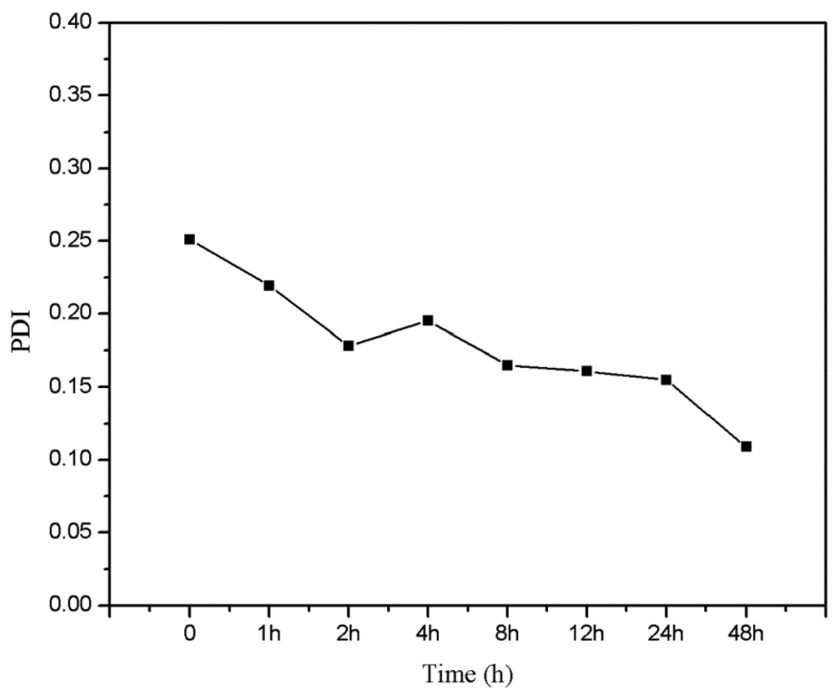

Fig. 7 Polydispersity index (PDI) of MSNR2 against faintly acidic conditions over $48 \mathrm{~h}$.
$<78.125 \mu \mathrm{g} \mathrm{mL}{ }^{-1}$, the viability of HeLa cells was about $100 \%$, indicating no obvious cytotoxicity. With further increases in the concentration to $156.25 \mu \mathrm{g} \mathrm{mL}{ }^{-1}$ of MSNRs, both MSNR2 and MSNR3 had a certain cytotoxicity, especially the cell viability of MSNR2 sharply decreased to $37.1 \%$, mainly due to the high specific surface area of MSNR2, which could induce the generation of more active oxygen species in tumor cells.

\subsection{Cellular oxidative stress level}

Cellular oxidative stress induced by MSNRs in HeLa cells was examined using FACS, as shown in Fig. 11. ROS generation was indicated by DCF fluorescence. The results showed the level of ROS of MSNR2 was significantly higher than that in MSNR1 and MSNR3. Besides, the MSNR2 caused a dose dependent increase in the ROS level of the HeLa cells. With increased concentration of MSNRs, the ROS level of MSNR1 cells also increased slowly, but the level in MSNR3 cells did not change significantly.

MSNs are an extraordinarily diverse family of inorganic nanomaterials, which are synthesized primarily from tetraalkoxysilanes or sodium silicate solutions. ${ }^{27}$ The $\mathrm{pH}$ of aqueous solution and template molecules used in MSN synthesis can control the pore size, pore ordering, morphology and reaction rate to form an ordered silicon dioxide matrix. ${ }^{28}$ In this work, new spiral MSNRs with different aspect ratios were synthesized 


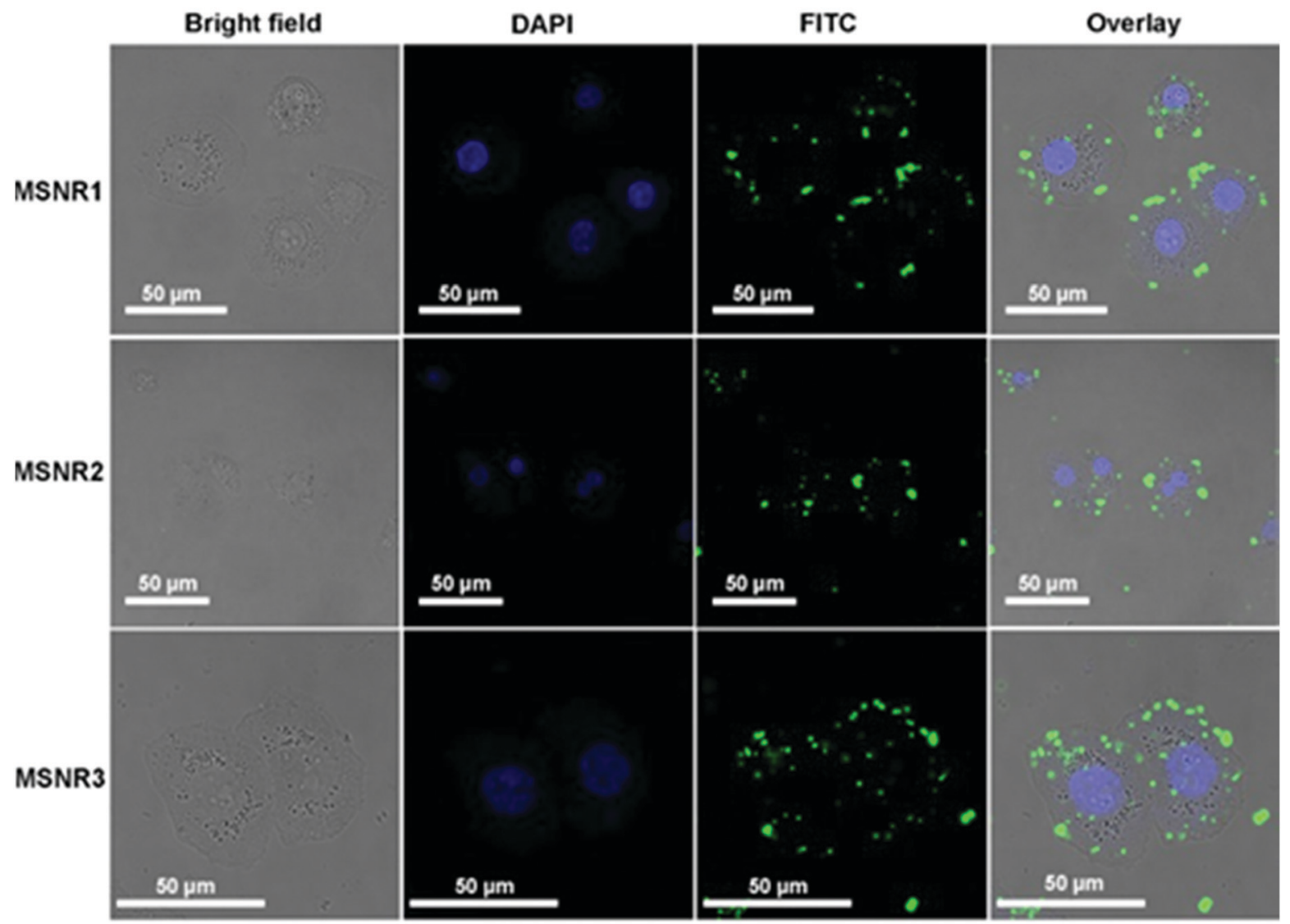

Fig. 8 Laser scanning confocal microscopy images of HeLa cells after the treatment of MSNRs for $2 \mathrm{~h}$.

through adjusting the dosages of $\mathrm{NH}_{3} \cdot \mathrm{H}_{2} \mathrm{O}$ by the modified Stöber method. ${ }^{29}$ These particles were denoted as MSNR1, MSNR2 and MSNR3 according to the $\mathrm{NH}_{3} \cdot \mathrm{H}_{2} \mathrm{O}$ dosage $(10 \mathrm{~mL}, 8 \mathrm{~mL}$ and $4 \mathrm{~mL}$ ). The characterizations of MSNRs were verified by TEM, FESEM, XRD and BET. The results showed that MSNRs had a $p 6 \mathrm{~mm}$ mesoscopic structure with a six square shape spiral morphology and two-dimensional six square arrangement of the channel. The ratio of the length to diameter of the MSNRs was 3.5 4.6, and 4.8, respectively. Furthermore, MSNRs with different aspect ratios had different specific surface area and pore volume. MSNR2 had the largest specific surface area $\left(1251 \mathrm{~m}^{2} \mathrm{~g}^{-1}\right)$ and pore volume $\left(1.05 \mathrm{~cm}^{3} \mathrm{~g}^{-1}\right)$; the BET surface areas of MSNR1 and MSNR3 were $1092 \mathrm{~m}^{2} \mathrm{~g}^{-1}$ and $1055 \mathrm{~m}^{2} \mathrm{~g}^{-1}$.

Nanomaterials have unique physico-chemical properties because of their small size and unusual structures. ${ }^{30-32}$ Despite their potential ability to move to cells, the toxicity of this new type of nanomaterial is still rarely reported. In vitro responses of HeLa cells were applied to study the biological safety of MSNRs. The ultrastructure of HeLa cells was incubated with MSNRs for $4 \mathrm{~h}$ and the results of LSCM showed that MSNRs could readily enter HeLa cells and be localized in the cytoplasm through endocytosis. MSNRs showed no significant toxicity to HeLa cells, as shown in the TEM images of cell ultrastructure. The HeLa cell viability of MSNRs was tested with the CCK-8 method at $24 \mathrm{~h}$. The results showed that all cell relative viability was almost $100 \%$ in all groups, even though MSNRs were at a high concentration of $160 \mu \mathrm{g} \mathrm{mL}^{-1}$, which indicated that MSNRs were biocompatible. With the concentration increased to $320 \mu \mathrm{g} \mathrm{mL}{ }^{-1}$, the cell viability of MSNR1 and MSNR2 decreased to $82.1 \%$ and $63.73 \%$. However, MSNR3 had almost no toxicity to HeLa cells, and the cell viability was $94.86 \%$. Interestingly, we found that cytotoxicity was positively related to the specific surface area of the MSNRs. The silanol groups ( $\mathrm{Si}-\mathrm{OH})$ on the surface of MSNRs could directly lead to cell membrane disruption and cytotoxicity, and the number and distribution of silanol groups could affect cytotoxicity. ${ }^{33-35}$ The cytotoxicity of MSNR2 was the largest because the surface area of MSNR2 was quite high with a large number of $\mathrm{Si}-\mathrm{OH}$. The CCK-8 results showed that the specific surface area of MSNRS might be a key factor to induce cytotoxicity.

The mechanism of induced cell death of MSNRs was further investigated by measuring the intracellular ROS level. Nonfluorescent probe DCFH-DA was delivered into HeLa cells before the ROS measurement. DCFH-DA could freely penetrate the plasma lemma into the cytoplasm and hydrolyze DCFH. ${ }^{36}$ By reacting with intracellular ROS, DCFH was converted into fluorescent matter DCF, which could be used to determine the amount of ROS. ${ }^{37}$ After $24 \mathrm{~h}$ exposure, the level of intracellular ROS in HeLa cells incubated with MSNR2 was remarkably higher than that with MSNR1, and MSNR3, which was consistent with the results of cytotoxicity. 

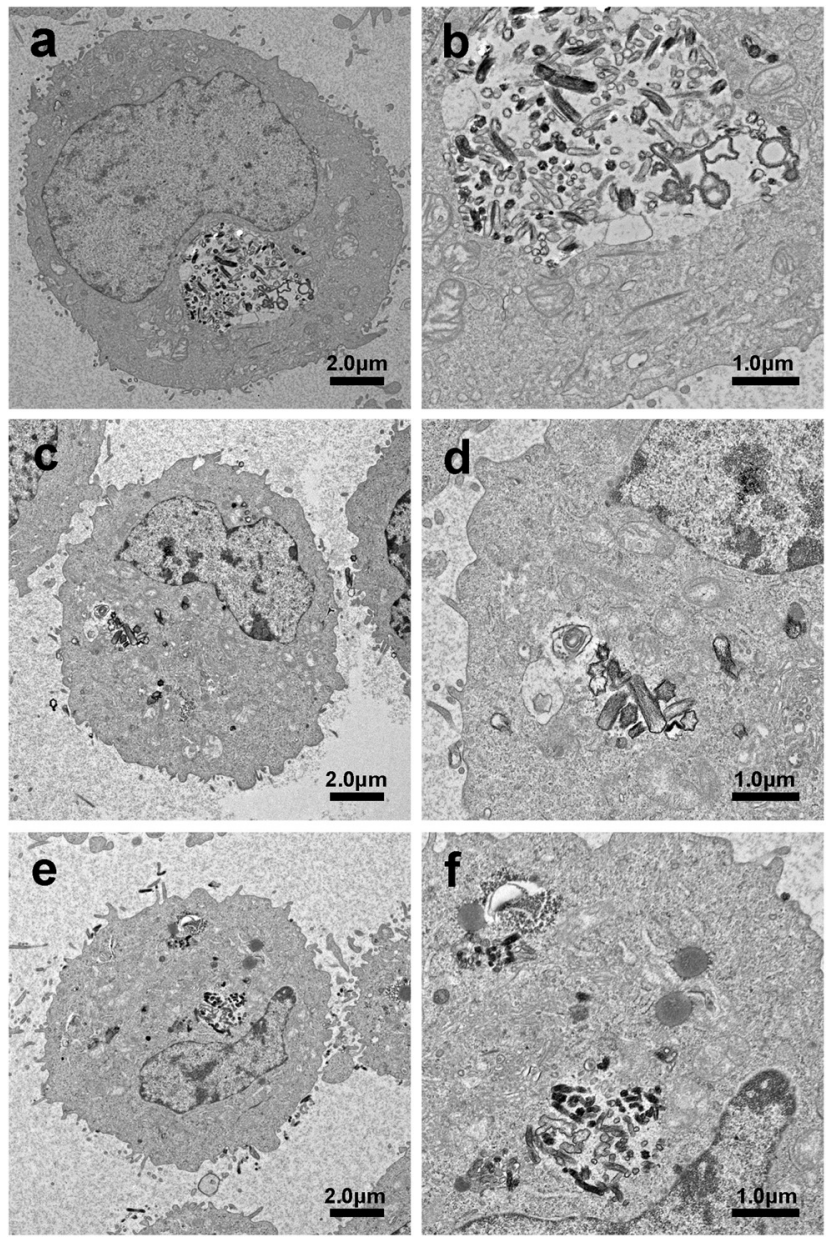

Fig. 9 Biological transmission electron microscopy images of HeLa cells after a $4 \mathrm{~h}$ exposure to MSNR1 ( $\mathrm{a}$ and b), MSNR2 ( $\mathrm{c}$ and d), and MSNR3 (e and f).

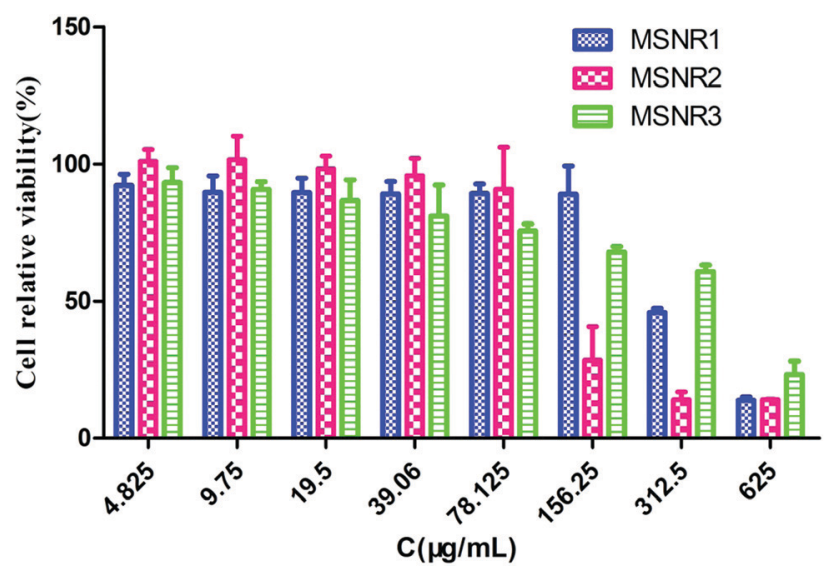

Fig. 10 Viability of HeLa cells incubated with different concentrations of MSNRs for $24 \mathrm{~h}$. The data are shown as the means \pm SD values obtained from three separate experiments.

In this section, the MSNRs showed remarkable cytotoxicity at higher concentrations, and the increased ROS yield induced

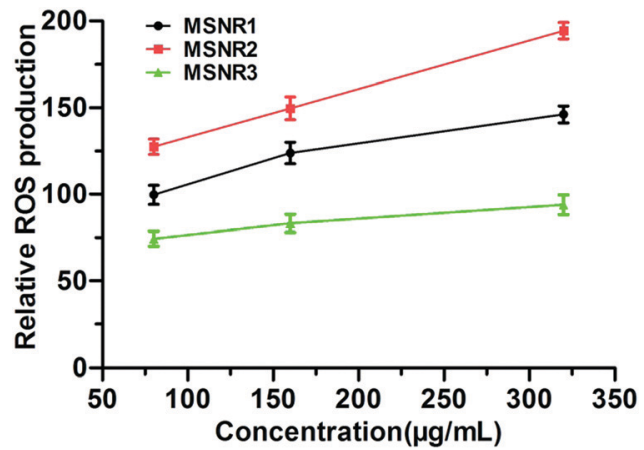

Fig. 11 The cellular reactive oxygen species levels of HeLa cells after $24 \mathrm{~h}$ exposure to different concentrations of MSNRs.

by MSNRs could be the major factor of this cytotoxicity, which was probably due to the specific surface area.

\section{Conclusions}

In this paper, three new spiral MSNRs with different aspect ratios were synthesized through adjustment of the dosages of $\mathrm{NH}_{3} \cdot \mathrm{H}_{2} \mathrm{O}$ by the modified Stöber method and the synthesized MSNRs were used to study their interactions with HeLa cells. MSNRs had periodical thread and narrower aperture size distribution. The mean pore sizes of MSNR1, MSNR2 and MSNR3 were $2.78 \mathrm{~nm}, 2.78 \mathrm{~nm}$ and $2.94 \mathrm{~nm}$, respectively. Also, DSL demonstrated benign hydrodynamic stability under faintly acidic conditions. As indicated by LSCM and TEM, MSNRs were found to be internalized by HeLa cells and were primarily localized in the cytoplasm, lysosomes and membranous vesicles. When the concentration of MSNRs was $160 \mu \mathrm{g} \mathrm{mL} \mathrm{m}^{-1}$, the viability of HeLa cells was about $100 \%$, and there was no obvious cytotoxicity. With further increases in the concentration to $320 \mu \mathrm{g} \mathrm{mL}{ }^{-1}$ of MSNRs, the cell viability of MSNR1 and MSNR2 decreased to $82.1 \%$ and $63.73 \%$, respectively, while MSNR3 was $94.86 \%$. Reactive oxygen species production was induced in the HeLa cells by the MSNRs, and reactive oxygen species generation could be the major factor causing cytotoxicity. The study indicated that the effect of MSNRs on HeLa cell viability and cellular oxidative stress was related to specific surface area and has great potential in clinical tumor therapy.

\section{Conflicts of interest}

There are no conflicts to declare.

\section{Acknowledgements}

This work was financially supported by a grant from the National Natural Science Foundation of China (21401214).

\section{References}

1 Y. Tang, F. Wang, C. Jin, H. Liang, X. Zhong and Y. Yang, Environ. Toxicol. Pharmacol., 2013, 36, 66-72. 
2 J. Yao, P. Li, L. Li and M. Yang, Acta Biomater., 2018, 74, 36-55.

3 B. G. Cha and J. Kim, Wiley Interdiscip. Rev.: Nanomed. Nanobiotechnol., 2018, e1515.

4 B. N. Ho, C. M. Pfeffer and A. Singh, Anticancer Res., 2017, 37, 5975-5981.

5 E. Piktel, K. Niemirowicz, M. Wątek, T. Wollny, P. Deptuła and R. Bucki, J. Nanobiotechnol., 2016, 14, 39.

6 H. Liang, C. Jin, Y. Tang, F. Wang, C. Ma and Y. Yang, J. Appl. Toxicol., 2014, 34, 367-372.

7 A. Watermann and J. Brieger, Nanomaterials, 2017, 7, 189.

8 Y. Zhou, G. Quan, Q. Wu, X. Zhang, B. Niu, B. Wu, Y. Huang, X. Pan and C. Wu, Acta Pharm. Sin. B, 2018, 8, 165-177.

9 S. B. Park, Y. Joo, H. Kim, W. Ryu and Y. Park, Mater. Sci. Eng., C, 2015, 50, 64-73.

10 L. Jia, Z. Li, J. Shen, D. Zheng, X. Tian, H. Guo and P. Chang, Int. J. Pharm., 2015, 89, 318-330.

11 G. F. Luo, W. H. Chen, Y. Liu, Q. Lei, R. X. Zhuo and X. Z. Zhang, Sci. Rep., 2014, 4, 6064.

12 M. A. Tölli, M. P. A. Ferreira, S. M. Kinnunen, J. Rysä, E. M. Mäkilä, Z. Szabó, R. E. Serpi, P. J. Ohukainen, M. J. Välimäki, A. M. R. Correia, J. J. Salonen, J. T. Hirvonen, H. J. Ruskoaho and H. A. Santos, Biomaterials, 2014, 35, 8394-8405.

13 X. Wang, Y. Zhang, W. Luo, A. A. Elzatahry, X. Cheng, A. Alghamdi, A. M. Abdullah, Y. Deng and D. Zhao, Chem. Mater., 2016, 28, 2356-2362.

14 C. Li, C. Ma, F. Wang, Z. Xi, Z. Wang, Y. Deng and N. He, J. Nanosci. Nanotechnol., 2012, 12, 2964-2972.

15 M. Niu, H. Zhong, H. Shao, D. Hong, T. Ma, K. Xu, X. Chen, J. Han and J. Sun, J. Nanosci. Nanotechnol., 2016, 16, 2313-2318.

16 M. Yu, L. Xu, F. Tian, Q. Su, N. Zheng, Y. Yang, J. Wang, A. Wang, C. Zhu, S. Guo, X. Zhang, Y. Gan, X. Shi and H. Gao, Nat. Commun., 2018, 9, 2607.

17 N. Li, Z. Wang, Y. Zhang, K. Zhang, J. Xie, Y. Liu, W. Li and N. Feng, Artif. Cells, Nanomed., Biotechnol., 2018, 1-15.

18 S. A. Shahin, R. Wang, S. I. Simargi, A. Contreras, L. Parra Echavarria, L. Qu, W. Wen, T. Dellinger, J. Unternaehrer, F. Tamanoi, J. I. Zink and C. A. Glackin, Nanomedicine, 2018, 14, 1381-1394.

19 J. Fan, G. Fang, X. Wang, F. Zeng, Y. Xiang and S. Wu, Nanotechnology, 2011, 22, 455102.
20 L. Lin, O. Elkashty, M. Ramamoorthi, N. Trinh, Y. Liu, G. Sunavala-Dossabhoy, T. Pranzatelli, D. G. Michael, C. Chivasso, J. Perret, J. A. Chiorini, C. Delporte and S. D. Tran, Oral Dis., 2018, 24, 1477-1483.

21 Z. Chen, M. Zhang, Y. Qiao, J. Yang and Q. Yin, Artif. Cells, Nanomed., Biotechnol., 2018, 1-7.

22 T. Hu, A. S. A. Qahtan, L. Lei, Z. Lei, D. Zhao and H. Nie, Bioact. Mater., 2017, 3, 48-54.

23 M. Ravinanthanan, M. N. Hegde, V. Shetty and S. Kumari, Contemp. Clin. Dent., 2018, 9, 255-259.

24 R. Riccò, S. Nizzero, E. Penna, A. Meneghello, E. Cretaio and F. Enrichi, J. Nanopart. Res., 2018, 20, 117.

25 Q. Yue, J. Li, W. Luo, Y. Zhang, A. A. Elzatahry, X. Wang, C. Wang, W. Li, X. Cheng, A. Alghamdi, A. M. Abdullah, Y. Deng and D. Zhao, J. Am. Chem. Soc., 2015, 137, 13282-13289.

26 B. Liu, L. Han and S. A. Che, Angew. Chem., Int. Ed., 2012, 51, 923-927.

27 H. Mekaru, J. Lu and F. Tamanoi, Adv. Drug Delivery Rev., 2015, 95, 40-49.

28 R. Roggers, S. Kanvinde, S. Boonsith and D. Oupický, AAPS PharmSciTech, 2014, 15, 1163-1171.

29 Y. Han, Z. Lu, Z. Teng, J. Liang, Z. Guo, D. Wang, M. Y. Han and W. Yang, Langmuir, 2017, 33, 5879-5890.

30 J. Jeevanandam, A. Barhoum, Y. S. Chan, A. Dufresne and M. K. Danquah, Beilstein J. Nanotechnol., 2018, 9, 1050-1074.

31 C. Rodriguez-Quijada, M. Sánchez-Purrà, H. de Puig and K. Hamad-Schifferli, J. Phys. Chem. B, 2018, 122, 2827-2840.

32 R. X. Zhang, J. Li, T. Zhang, M. A. Amini, C. He, B. Lu, T. Ahmed, H. Lip, A. M. Rauth and X. Y. Wu, Acta Pharmacol. Sin., 2018, 39, 825-844.

33 P. J. A. Borm, P. Fowler and D. Kirkland, Part. Fibre Toxicol., 2018, 15, 23.

34 D. Ni, D. Jiang, E. B. Ehlerding, P. Huang and W. Cai, Acc. Chem. Res., 2018, 51, 778-788.

35 A. M. Schrader, J. I. Monroe, R. Sheil, H. A. Dobbs, T. J. Keller, Y. Li, S. Jain, M. S. Shell, J. N. Israelachvili and S. Han, Proc. Natl. Acad. Sci. U. S. A., 2018, 115, 2890-2895.

36 F. Wang, C. Jin, H. Liang, Y. Tang, H. Zhang and Y. Yang, Environ. Toxicol. Pharmacol., 2014, 37, 656-661.

37 J. Zhan, Z. Ma, D. Wang, X. Li, X. Li, L. Le, A. Kang, P. Hu, L. She and F. Yang, Int. J. Nanomed., 2017, 12, 2733-2748. 